\title{
Antagonismos discursivos nas hashtags \# MARQUeTEIROSDOJAIR E \#BOLSOLÃo No TWITTER NAS ELEIÇÕES DE 2018 NO BRASIL: CONTRIBUIÇÕES DA ANÁLISE DE REDES SOCIAIS À SOCIOLOGIA DIGITAL
}

\author{
Otávio VINHAS* \\ Nilton SAINZ** \\ Raquel RECUERO**
}

\begin{abstract}
RESUMO: Este artigo tem como objetivo analisar a formação de antagonismos discursivos nas hashtags \#bolsolão e \#marqueteirosdojair, no contexto polarizado das Eleições de 2018 no Brasil. Em contribuição aos desafios teóricometodológicos da Sociologia Digital, partimos de uma abordagem interdisciplinar, articulando a Teoria do Discurso de Ernesto Laclau e Chantal Mouffe à Análise de Redes Sociais na internet. A partir disso, coletamos um total de 26.548 tweets da API do Twitter através do software NodeXL Pro. Assim, investigamos as duas hashtags selecionadas através de uma análise de contingência, conforme as métricas da modularidade e do grau de intermediação. Desse modo, verificamos quais os sentidos encadeados na formação das duas hashtags, descrevendo como estão articulados segundo as lógicas de equivalência e de diferença. Concluímos que a nossa proposta teórico-metodológica permite observar a polarização política como um fenômeno dinâmico, contingente e contraditório no social, associado à emergência de antagonismos discursivos.
\end{abstract}

PALAVRAS-CHAVE: Sociologia Digital. Teoria do Discurso. Twitter. Análise de Redes Sociais; Eleições 2018.

\footnotetext{
UFPel - Universidade Federal de Pelotas - Programa de Pós-Graduação em Sociologia - Pelotas RS - Brasil. 96010-160 - otavio.vinhas@gmail.com. https://orcid.org/0000-0002-0226-4371.

* UFPR - Universidade Federal do Paraná - Programa de Pós-Graduação em Ciência Política Curitiba - PR - Brasil. 80060-150 - sainznilton@gmail.com. https://orcid.org/0000-0002-3957-2714.

*** UFRGS - Universidade Federal do Rio Grande do Sul - Programa de Pós-Graduação em Comunicação - Porto Alegre - RS - Brasil. 90035-007 - raquelrecuero@gmail.com. https://orcid. org/0000-0002-7417-9782.
} 


\section{Introdução}

A intenção deste trabalho consiste em investigar a formação de antagonismos discursivos (LACLAU \& MOUFFE, 2015) em interações sociais no Twitter no contexto das Eleições Presidenciais de 2018 no Brasil. Através de um diálogo interdisciplinar entre as áreas da Sociologia, Ciência Política e Comunicação Social, propomos uma abordagem original, centrada na articulação da Teoria do Discurso de Ernesto Laclau e Chantal Mouffe, aos estudos em Análise de Redes Sociais na internet e à Análise de Contingência. Nessa perspectiva, procuramos estabelecer uma contribuição à Sociologia Digital a partir do seguinte questionamento: Como estão articuladas discursivamente as hashtags \#bolsolão e \#marqueteirosdojair, conforme as lógicas de equivalência e de diferença, segundo a Teoria do Discurso de Ernesto Laclau e Chantal Mouffe?

Partimos do pressuposto de que o Twitter, como um site de rede social (BOYD \& ELLISON, 2007), proporciona a emergência de conversações e, consequentemente, a formação de discursos. Dessa forma, delimitamos a nossa investigação às interações sociais no Twitter em torno da matéria de título "Empresários bancam campanha contra o PT pelo WhatsApp", divulgada pelo jornal Folha de São Paulo no dia 18 de outubro de 2018 (MELLO, 2018), articuladas nas hashtags \#bolsolão e \#marqueteirosdojair. O conteúdo da matéria trata a respeito de uma prática, promovida por empresários favoráveis à campanha de Jair Bolsonaro à presidência da república, de contratar agências que prestam serviços de "disparo em massa" de mensagens em grupos no WhatsApp. Segundo a reportagem, a prática relatada é considerada ilegal pelo ordenamento jurídico brasileiro. Naquele momento, a matéria passou a ser um dos principais assuntos comentados no Twitter, a partir de interações articuladas principalmente nas hashtags "\#bolsolão" e "\#marqueteirosdojair".

$\mathrm{O}$ trabalho encontra-se organizado em três partes. Na primeira parte, traçamos um breve panorama acerca do estabelecimento da Sociologia Digital como uma subdisciplina do conhecimento, apontando possíveis contribuições que a Análise de Redes Sociais na internet pode oferecer aos desafios sociológicos do século XXI. $\mathrm{Na}$ segunda parte, explicamos a nossa lente teórico-epistemológica direcionada a observar os fenômenos de polarização e da emergência de antagonismos discursivos nas interações sociais no Twitter, com base em conceitos advindos da Teoria do Discurso de Ernesto Laclau e Chantal Mouffe. Na terceira parte, apresentamos os procedimentos metodológicos, a análise dos dados e as respostas a que chegamos frente à problemática de pesquisa. 
Antagonismos discursivos nas hashtags \#marqueteirosdojair e \#bolsoläo no Twitter nas eleiçôes de 2018 no Brasil: contribuiçóes da análise de redes sociais à sociologia digital

\section{Contribuições teórico-metodológicas da Análise de Redes Sociais para a Sociologia Digital}

As formas pelas quais, atualmente, as mobilizações e as articulações políticas vêm sendo construídas têm instigado pesquisadores das ciências sociais a buscar modelos de explicação que contemplem as dinâmicas sociais produzidas pela mediação do digital. Por se tratar de uma pretensão eminentemente complexa, a busca pela interdisciplinaridade nas ciências sociais mostra-se imprescindível para o enfrentamento desses desafios inescapáveis à pesquisa do século XXI. Nesse contexto, abordamos as articulações políticas desempenhadas no on-line a partir de uma construção teórico-metodológica propícia ao ofício da Sociologia Digital. Para isso, propomos um estudo sobre uma questão central, o discurso na mediação do Twitter, constituído através do uso de marcadores como as hashtags, de uma operacionalização do instrumental analítico-observacional da Análise de Redes Sociais na internet e da Análise de Contingência, para investigar a configuração de um cenário de polarização política, de acordo com aportes advindos da teoria social contemporânea.

A Sociologia Digital surge como uma subdisciplina voltada a compreender e a assimilar o uso das tecnologias digitais na pesquisa sociológica (WYNN, 2009). Em face às novas problemáticas suscitadas pela imersão das tecnologias digitais no cotidiano, a Sociologia Digital é vista como um convite ao repensamento de teorias e métodos consolidados anteriormente à era pré-digital (SELWYN, 2019). Por conseguinte, a tarefa da Sociologia Digital consiste em repensar, a partir de modelos teórico-metodológicos novos ou renovados, as configurações entre público/ privado, tradição/modernidade, rural/urbano e as demais fronteiras responsáveis por caracterizar as relações sociais (MISKOLCI, 2016). Sob esse direcionamento, Lupton (2015) resume os desafios da Sociologia Digital a quatro frentes distintas, quais sejam: 1) a utilização de ferramentas digitais para compartilhar pesquisas e a aproximar pesquisadores; 2) a análise dos usos sociais e das funções das mídias digitais na criação e reprodução de instituições e estruturas sociais; 3 ) a construção de métodos qualitativos e quantitativos para a análise de dados digitais; 4) a reflexão sobre as tecnologias digitais de acordo com as teorias sociais e culturais já existentes.

$\mathrm{Na}$ esteira dessa interação entre métodos e teorias, o horizonte da Sociologia Digital passa a envolver uma constante (re)leitura do fenômeno social, no que tange às interseções conceituais entre tecnologia, sociabilidade e conhecimento (MARRES, 2017). Conforme aponta Nascimento (2016), para lidar com este cenário, torna-se necessário um esforço reflexivo, no sentido de integrar os métodos de coleta e de organização de dados produzidos digitalmente ao aparato teórico e epistemológico da Sociologia. Diante dessa discussão, Marres e Gerlitz (2016) propõem a formulação do que chamam de métodos de interface. Esse conceito 
denota que não há a necessidade de ferramentas digitais serem desenvolvidas especificamente para atender aos preceitos disciplinares de pesquisadores sociais e/ou culturais. Contrariamente a isso, campos sociais do conhecimento podem observar ferramentas digitais em geral como "oportunidades epistêmicas", abertas a experimentações metodológicas dispostas a encontrar formas de responder a questionamentos socialmente relevantes (MARRES; GERLITZ, 2016, p.27-30). Na perspectiva proposta pelas autoras, métodos já consolidados cientificamente podem ser beneficiados, por exemplo, pelas $A P I \mathrm{~s}^{1}$ proporcionadas pelas plataformas digitais $^{2}$ - como é o caso do Twitter -, desde que verificadas as capacidades e as limitações de tais métodos para observar contextos sociais (MARRES; GERLITZ, 2016).

Neste sentido, nossa proposta é aproveitar dados obtidos pela API do Twitter para discutir o fenômeno dos antagonismos discursivos a partir da perspectiva da Teoria do Discurso de Ernesto Laclau e Chantal Mouffe (2015), estabelecendo de uma combinação teórico-metodológica que acene à essa proposta. Deste modo, fazemos uma discussão através de uma combinação de análise de redes (ARS) e uma análise de contingência - que é parte da Análise de Conteúdo, segundo Bardin (1977) - como métodos pertinentes às propostas e aos desafios relativos à Sociologia Digital.

Um dos métodos que vem se destacando na construção e/ou na adaptação às ferramentas digitais é o da Análise de Redes Sociais (ARS) (RECUERO, 2017). Embora encontre as suas raízes na década de 1930, a partir da Sociometria de Jacob Moreno, é também uma abordagem utilizada para compreender as interações sociais constituídas digitalmente (FRAGOSO; RECUERO; AMARAL, 2016). No que tange às explorações empíricas, ao longo do Século XX, as técnicas desenvolvidas pela ARS, de coleta, de processamento, e de organização de dados, mostram-se proficuamente aplicáveis a objetos de pesquisa observados no âmbito digital (RECUERO; BASTOS; ZAGO, 2015). Assim, quando voltada ao estudo das interações sociais na internet, a ARS não representa um método propriamente novo, mas uma abordagem possível de ser revigorada, principalmente à luz da emergência de novas ferramentas digitais, bem como de novas problemáticas à pesquisa social, sobretudo à Sociologia Digital (MARRES, 2017). O intuito da ARS consiste em estudar os componentes, as conexões e/ou os princípios estruturais imbricados às formas como se conectam os componentes de uma rede (NEWMAN, 2010). Em princípio, trata-se de uma abordagem orientada para uma análise estruturalista, no

\footnotetext{
1 A API (em português: Interface de Programação de Aplicações) de uma plataforma é uma interface intermediária, destinada a facilitar a utilização de certos recursos da plataforma que normalmente não são disponíveis ao usuário comum.

2 Nesse sentido, referimos-nos ao Twitter de acordo com o conceito de "plataformas digitais" de Tarleton Gillespie (2010), tendo em vista, além da sua acepção figurativa como uma plataforma voltada à interações, as suas características computacionais, arquitetônicas e políticas.
} 
sentido da busca por identificar padrões responsáveis por moldar ou influenciar um determinado conjunto de relações (FRAGOSO; RECUERO; AMARAL, 2016). Todavia, embora possua um método com fundamentos consolidados, as técnicas e os aportes investigativos da ARS são amplamente adotados a partir de perspectivas interdisciplinares, em combinação a conceitos e teorias advindas de diferentes campos do conhecimento (WASSERMAN; FAUST, 1994).

Aplicada à pesquisa na internet, a ARS possui a prerrogativa de expor e publicar as redes sociais dos atores emergentes na estrutura de uma rede, constituída por conversações em sites e/ou plataformas digitais (RECUERO; BASTOS; ZAGO, 2015). Para isso, são observadas as relações entre os nós (componentes de uma rede) e as arestas (conexões), de modo a investigar os nexos de causação e/ou de correlação presentes na posição de um ou mais nós, em combinação à estrutura geral de uma rede (RECUERO, 2017). Segundo essa lógica, o objetivo da ARS consiste em permitir que relações complexas sejam sintetizadas e estruturadas em um modelo de representação inteligível, capaz de ser observado através de diferentes vertentes teórico-epistemológicas.

Ao mesmo tempo, a análise de contingência é uma perspectiva de análise de conteúdo (BARDIN, 1977, p.18) que se refere à "análise das co-ocorrências de temas ou conceitos, ou exclusões, presentes no material de análise" e encontra suas raízes nos trabalhos de Osgood (1959). Essa perspectiva visa compreender a estrutura das relações de um determinado discurso através da frequência de determinadas associações nos dados. Como parte da Análise de Conteúdo, utiliza suas premissas de classificação e organização dos dados.

\section{Antagonismos discursivos e a polarização política no Twitter}

\subsection{A emergência de antagonismos discursivos segundo a Teoria do Discurso}

Eventos sociopolíticos recentes, a exemplo das Eleições de 2016 nos Estados Unidos, do Brexit no Reino Unido e das Eleições de 2018 no Brasil, têm se caracterizado por polarizações políticas, principalmente a partir daquilo que se observa nas interações sociais na internet (BENKLER; FARIS; ROBERTS, 2018). A formação de tais contextos ocorre de maneira complexa, uma vez que há um intrincamento permanente entre o que ocorre no real e os aspectos específicos atinentes às relações constituídas no digital. Observamos, em relação a essa problemática, que os aportes teórico-epistemológicos da Teoria do Discurso de Ernesto Laclau e Chantal Mouffe (2015) representam ferramentas metodológicas cujo potencial interdisciplinar mostra-se imprescindível para estudos empíricos na 
América Latina (COSTA; FREITAS; MERLIN, 2019). A partir dessa perspectiva, centramos-nos na ideia de antagonismos discursivos - e demais conceitos adjacentes, como articulações discursivas, ponto nodal, lógica de equivalência e lógica de diferença - para observar a emergência de um contexto polarizado, como parte de um social relacional, indeterminado e interconectado (LACLAU; MOUFFE, 2015).

A Teoria do Discurso (TD), como lente teórico-epistemológica voltada à descrição do cenário sociopolítico contemporâneo, estabelece o conceito de discurso como uma unidade complexa, constituída por palavras e ações (BURITY, 2014). Na perspectiva de Laclau e Mouffe (2015), o discurso é uma construção contínua, articulada em um social radicalmente indeterminado, caracterizado pela contingência e pela precariedade das relações. Em razão disso, o social é visto como uma dimensão na qual não existe uma essência teleológica e/ou estática capaz de representar o mundo através de todos os elementos que o compõem (LACLAU; MOUFFE, 2015). Assim, o discurso corresponde a um conjunto de regras, capaz de constituir uma unidade contingente e precária, a partir da atribuição de regularidade a elementos ${ }^{3}$ em dispersão (LACLAU, 2005; LACLAU; MOUFFE, 2015).

A construção do discurso, da forma entendida por Laclau e Mouffe (2015), envolve o que é chamado de articulações discursivas. Em contraste a uma lógica habermasiana, pautada pela racionalidade das relações, bem como pela possibilidade de formação de consensos intersubjetivos (HABERMAS, 1989), o discurso não é articulado no plano da retórica, mas no âmbito afetivo (LACLAU, 2005). Por conseguinte, a partir de uma visão da política como uma instância ligada aos desejos e ao poder, tais articulações discursivas passam a emergir no social a partir de associações entre afetos compartilhados (MOUFFE, 2005). Nesse sentido, a noção de articulações discursivas - mesmo ao se pensar sobre as interações digitais torna a ideia racional de esfera pública (HABERMAS, 1989), por exemplo, uma "impossibilidade conceitual" (LACLAU; MOUFFE, 2015, p.46) na medida em que os vínculos afetivos tendem a criar conflitos de um "nós" contra um "eles" (MOUFFE, 2005).

As articulações discursivas são constituídas, portanto, em contextos de constantes disputas. Conforme Mouffe (2013), os preceitos para a emergência de uma articulação discursiva são as diferenças em comum identificadas por elementos discursivamente articulados em relação a outros. A isso, Laclau e Mouffe (2015) referem-se como "exterior constitutivo", isto é, a observação de uma identidade outra (eles) contra a qual uma articulação (nós) emerge, na tentativa de dominar o campo da discursividade. Dessa forma, as articulações discursivas não seguem um princípio subjacente de representação (de classe, por exemplo), mas de articulações no sentido

\footnotetext{
3 Um elemento, na ótica da TD, corresponde a uma diferença que não se encontra discursivamente articulada (LACLAU; MOUFFE, 2015).
} 
da prática e da luta política, sempre de modo contingente e precário (LACLAU; MOUFFE, 2015). Nessa lógica, o aspecto relacional, atinente às disputas que fundamentam a emergência de articulações discursivas, impossibilitam a construção de uma totalidade ${ }^{4}$, uma vez que a emergência de um discurso está sempre ligada a uma articulação contra um inimigo em comum (LACLAU; MOUFFE, 2015).

Essa impossibilidade de fechamento de uma ordem total - isto é, de uma sociedade, tal como na concepção da TD - ocorre pelos limites estabelecidos pela dinâmica antagonística do social (LACLAU; MOUFFE, 2015). O conceito de antagonismo consta como o "centro da atual relevância" do enfoque adotado por Laclau e Mouffe (2015, p.42). Através da observação de antagonismos discursivos, é possível não apenas descrever as articulações e as causas que abrangem uma relação antagonística, mas definir as contradições envolvidas em antagonismos, as quais fazem-se presentes no social (LACLAU; MOUFFE, 2015). Dessa forma, podemos pensar a emergência de antagonismos analogamente ao que o senso comum entende como "duas faces de uma mesma moeda", ao invés de associar o conceito à mera presença de duas forças distintas em oposição (LACLAU; MOUFFE, 2015). Assim, conforme Laclau e Mouffe (2015, p.204), o antagonismo pode ser definido como "uma relação na qual se mostram os limites de toda objetividade", de modo que a inescapabilidade da presença antagonística associada a um inimigo outro é o que impede a sociedade de compor uma realidade objetiva.

A constituição de antagonismos discursivos pressupõe dinâmicas de articulação e de repulsão entre elementos heterogêneos, conforme o que Laclau (2005, p.200) chama de "lógicas de equivalência e de diferença". No tocante a isso, uma articulação é composta mediante o encadeamento entre elementos aleatórios no social, os quais passam a compor momentos de uma unidade (discurso) contingente, através do reconhecimento de uma identificação negativa mútua em relação a seu exterior constitutivo (inimigo em comum) (LACLAU, 2005). Sob essa perspectiva a lógica de equivalência corresponde a uma forma de articulação baseada na subversão ou na supressão parcial de diferenças, em prol de combater um inimigo em comum. Já a lógica de diferença está relacionada à constatação de tais distinções, as quais são deixadas de lado momentaneamente, como condição de possibilidade para haja uma articulação entre diferentes (LACLAU, 2014).

A circunstância em que tais lógicas são articuladas envolve a emergência daquilo que Laclau chama de ponto nodal (LACLAU; MOUFFE, 2015). Esse conceito corresponde a uma plataforma de inscrição, através da qual elementos conseguem, contingentemente, se articular conjuntamente (LACLAU, 2005). Através de um ponto nodal, elementos heterogêneos constroem cadeias de equivalências,

\footnotetext{
4 A própria noção de sociedade, por se tratar de uma organização uníssona e, portanto, consensual, não existe, segundo a ótica de Laclau e Mouffe (2015).
} 
mediante as quais são articuladas as lógicas de equivalência e de diferença, no sentido de transcender as diferenças compartilhadas entre si para se organizar no teor de combater um inimigo em comum (LACLAU, 2014). Nesse sentido, conforme apontam estudos anteriores, pontos nodais podem ser verificados nas interações digitais, seja através de menções de certas palavras/conceitos ou mesmo de hashtags (COSTA, 2014), caracterizando contextos de polarização política observados como antagonismos plataformizados (FARKAS; SCHOU; NEUMAYER, 2018).

\subsection{A formação de contextos de polarização política no Twitter}

As características das interações sociais desempenhadas em plataformas digitais representam um campo fértil para a emergência de antagonismos discursivos, contribuindo para a formação de cenários polarizados (GERBAUDO, 2018). Conforme afirma Herring (2001), as lógicas implicadas na ótica do discurso também se mostram presentes nas interações via computadores ou smartphones. Isso pode ser verificado na medida em que as articulações discursivas não emergem, necessariamente, a partir do conteúdo de redes de conversações, mas por padrões meta-comunicativos, responsáveis por orientar a ordenação de um discurso em torno de um inimigo em comum (CESARINO, 2020).

Os contextos político-eleitorais recentes de polarização política, tal como se observa no Brasil em 2018 (SOARES; RECUERO; ZAGO, 2019; MIGUEL, 2019), não raro, são associados à hipótese da formação de filtros-bolha em plataformas digitais (PARISER, 2011). Conforme Pariser (2011), um filtro-bolha representa um espaço em que apenas determinados conteúdos conseguem circular. De acordo com Bruns (2019), porém, ao se levantar a hipótese dos filtros-bolha, o debate sobre polarização política no social tende a ser reduzido a uma questão de mera dieta midiática, em uma perspectiva na qual contextos polarizados são vistos como consequência apenas das ações de algoritmos de personalização. Dessa forma, esse entendimento tende a dissociar o problema da polarização de questões sociais de maior complexidade, as quais indicam que contextos polarizados também se encontram associados à emergência de populismos e/ou sentimentos sociais radicalizados transpostos à política (BRUNS 2019). Nesse sentido, Bruns (2019) aponta que plataformas genéricas, como o Twitter e o Facebook tendem, justamente, a permitir a lógica contrária àquela dos filtros-bolha, ou seja, propiciando que determinados grupos observem ativamente com quais conteúdos o "outro lado" costuma interagir.

As maneiras específicas pelas quais ocorrem as interações on-line, de todo modo, mostram-se consoantes aos aspectos técnico-arquitetônicos de qualquer ambiente digital. Segundo Van Dijck (2013), a principal característica de uma 
plataforma digital é o intercâmbio entre a participação social de usuários e os recursos de conectividade disponibilizados pela interface de uma plataforma. Isso denota que tais plataformas tratam-se de serviços oferecidos na internet, caracterizados pela dinâmica estabelecida entre as funcionalidades colaborativas e/ou amigáveis disponibilizadas aos usuários e a exploração comercial dos dados produzidos nas interações sociais, mediante a codificação de informações para a forma de protocolos quantificáveis - "amigos", "likes", "seguidores" (VAN DIJCK, 2013).

No caso do Twitter, as hashtags exercem função essencial para o desenvolvimento das interações sob a forma de redes sociais on-line. Conforme afirma Salazar (2017), uma hashtag é uma superfície digital que exerce a função de encapsular diversas mensagens circunscritas sobre ela. Essa dinâmica faz com que a emergência de uma hashtag, na medida em que passa a representar uma plataforma para a propagação de determinadas mensagens, também se torne um evento por si próprio (SALAZAR, 2017). Assim, uma hashtag conecta mensagens (tweets), dando-lhes contexto ou complemento do que é dito. Nesse sentido, as dinâmicas interacionais constituídas em torno de uma hashtag tendem a seguir um continuum, pelo qual os sentidos representados sob uma determinada cerquila (\#) tendem a ser modificados ao longo do tempo, na medida em que são atraídas novas mensagens (BRUNS; BURGESS, 2011).

Por se tratar de um site de rede social (BOYD; ELLISON, 2007), as interações no Twitter são caracterizadas por três aspectos: a) pela visibilidade das conversações; b) pela criação de perfis públicos; c) pela formação de redes sociais on-line. Esses aspectos favorecem que as interações entre os usuários do Twitter sejam desempenhadas autorreflexivamente, isto é, de modo que os usuários estejam autocientes do teor performático contido nas interações (PAPACHARISSI, 2012). Segundo Papacharissi (2009), ao passo que a visibilidade das conversações tende a promover interações de tendências narcísicas, também permite que determinadas mensagens, principalmente aquelas de caráter considerado subversivo, possam ser amplificadas para públicos maiores. Isso favorece, sobretudo, que o Twitter seja visto um espaço de constantes disputas de sentidos e de competições por visibilidade (RECUERO, 2018) e, por conseguinte, como um ambiente interacional disposto à formação de antagonismos discursivos, segundo o viés da TD (LACLAU; MOUFFE, 2015).

\section{Proposta de Análise: Análise de contingência e Análise de Redes Sociais frente à Teoria do Discurso}

Com o objetivo de analisar a formação de antagonismos discursivos no Twitter através de uma proposta metodológica que possa também contribuir 
para a Sociologia Digital, o foco de investigação direciona-se às características e às diferenças relacionais entre as hashtags \#marqueteirosdojair e \#bolsolão, entendendo-as como articulações discursivas formadas em oposição. Para tanto, desempenhamos a coleta de um total de 26.548 tweets, sendo 5.217 correspondentes à "\#marqueteirosdojair" e 21.331 tweets que mencionaram a hashtag "\#bolsolão". Estas hashtags apareceram entre os assuntos mais comentados no Twitter (trending topics) durante o dia 18 de outubro de 2018, data da publicação da matéria "Empresários bancam campanha contra o PT pelo WhatsApp" no jornal Folha de São Paulo (MELLO, 2018). Em razão da proeminência destas hashtags naquele contexto, observamos a possibilidade de que ambas emergiram como discursos reciprocamente organizados, em combate um ao outro ou, por outras palavras, como articulações antagônicas e relacionais (LACLAU; MOUFFE, 2015). A partir disso, ao descrevermos as relações antagonísticas formadas nos discursos articuladas nas duas hashtags analisadas, observamos como a ARS, articulada junto à TD de Laclau e Mouffe (2015), pode fornecer bases teórico-metodológicas à Sociologia Digital.

\subsection{Coleta de dados}

O procedimento de coleta dos dados foi desempenhado com o auxílio do software NodeXL Pro 5 . Trata-se de um programa de computador capaz de realizar processos de coleta no Twitter de forma automática e retroativa, por meio do acesso à API da plataforma, até um limite de cerca de 18.000 (dezoito mil) tweets por coleta.

Ao todo, foram coletados um total de 26.548 tweets, emitidos entre os horários das $15 \mathrm{~h} 32 \mathrm{~min}$ às $19 \mathrm{~h} 53 \mathrm{~min}$ para \#marqueteirosdojair e no período das $16 \mathrm{~h} 57 \mathrm{~min}$ às $18 \mathrm{~h} 06 \mathrm{~min}$ para \#bolsolão. Visto que uma das hashtags (\#marqueteirosdojair) apresentou um número de tweets relativamente menor do que a outra (\#bolsolao) na primeira coleta, efetuamos uma coleta adicional para aproximar a diferença, conforme ilustramos no Quadro 1.

Quadro 1: corpus de análise

\begin{tabular}{|l|c|c|}
\hline \multicolumn{1}{|c|}{ Data e horário das coletas } & Hashtag & Número de Tweets \\
\hline 18/10/2018 às $17 \mathrm{~h} 50 \mathrm{~min}$ & \#bolsolão & 21.331 \\
\hline 18/10/2018 às $17 \mathrm{~h} 05 \mathrm{~min}$ e às $19 \mathrm{~h} 15 \mathrm{~min}$ & \#marqueteirosdojair & 5.217 \\
\hline
\end{tabular}

Fonte: Elaboração própria.

\footnotetext{
5 O NodeXL é uma aplicação open source para o programa Microsoft Excel, destinada a coletar e organizar dados de plataformas digitais. Site: https://nodexl.com/.
} 
Uma vez constituído o corpus, passamos a análise dos dados. Neste processo, trabalhamos inicialmente com a análise de contingência, cujos procedimentos são análogos aos da Análise de Conteúdo. Com o auxílio de software observamos as ocorrências de conjuntos de palavras em cada unidade (tweet). O sistema, inicialmente conta o número de vezes em que cada léxico apareceu em um tweet. $\mathrm{Na}$ primeira fase, selecionamos os 20 (vinte) léxicos mais frequentes em cada hashtag. Nesta etapa, independentemente da frequência, léxicos pouco significativos, como pronomes, artigos e preposições são eliminados do corpus. A partir desta primeira classificação, passamos ao segundo passo: A aglutinação de léxicos semelhantes em um mesmo conceito, a codificação. Nesta fase, palavras com sentido semelhantes ou palavras idênticas, porém escritas de modo diferente são aglutinadas em um mesmo código (por exemplo, Brasil, País, Brazil, etc.). Finalmente, no terceiro passo, obtivemos as co-ocorrências desses códigos, permitindo a construção de uma rede de conceitos que estão conectados entre si, conforme os dados obtidos nos tweets. A partir desta fase, utilizamos, então, métricas de Análise de Redes para indicar a importância dessas conexões e a centralidade dos conceitos no corpus, de modo a permitir identificar as articulações discursivas emergentes nas hashtags.

Assim, aplicamos a análise de contingência para tentar verificar as correlações formadas entre redes compostas por palavras/conceitos e tentar identificar a relacionalidade entre as palavras/conceitos que emergiram sob as hashtags \#marqueteirosdojair e \#bolsolão.

A organização e classificação dos dados, de acordo com os dois métodos mencionados, é efetuada através do software Gephi ${ }^{6}$. Nesse procedimento, aplicamos dois tipos de métricas da ARS para auxiliar a nossa análise: a métrica da modularidade e a métrica do grau de intermediação.

A métrica da modularidade calcula os índices de co-associações exibidos pelos nós de uma rede. Por meio de um algoritmo que calcula a força de atração entre os nós - force atlas (BLONDEL; GUILLAUME; LAMBIOTTE; LEFEBVRE, 2008) -, essa métrica define as posições dos nós, bem como das suas respectivas conexões, estabelecendo diferentes agrupamentos em uma rede, conforme o grau de redundância apresentado pelos nós de uma rede. Dessa forma, para o objetivo do presente artigo, a aplicação da métrica da modularidade nas redes analisadas permite observar quais os diferentes discursos inscritos articulados sob uma determinada hashtag no Twitter, no sentido de uma lógica de diferença (LACLAU; MOUFFE, 2015).

A métrica da intermediação calcula o índice da centralidade de um nó em uma rede. Isso permite identificar quais os nós que exerceram a função de uma ponte na holística dos componentes de uma rede (RECUERO, 2017). No sentido de um

6 Gephi. https://gephi.org/. 
cálculo voltado a investigar a formação de discursos, a métrica da intermediação expõe quais são os principais conceitos/palavras responsáveis por interconectar e, assim, compor uma unidade entre os diferentes elementos presentes um determinado discurso. Dessa forma, sob uma perspectiva vinculada à TD, o grau de intermediação dos nós de uma rede permite verificar quais conceitos/palavras articularam-se como pontos nodais para a construção de articulações discursivas em uma hashtag, segundo as lógicas de equivalência e de diferença (LACLAU; MOUFFE, 2015).

Nos grafos, os cálculos gerados pelas métricas utilizadas estará ilustrado da seguinte forma:

a) Métrica da Modularidade: agrupamentos de palavras, definidos por uma cor respectiva;

b) Métrica da Intermediação: tamanho dos nós que compõem a rede, ou seja, quanto maior o nó, mais elevado o grau de intermediação;

\subsection{Análise dos Dados}

Iniciamos a análise pela hashtag \#bolsolão, observando o grafo correspondente às redes de interação que se articularam contrariamente a Jair Bolsonaro, de acordo com a ilustração abaixo (Figura 1). No grafo, os conceitos com maior frequência individual no corpus aparecem em tamanho maior. Do mesmo modo, as conexões mais fortes entre eles aparecem mais demarcadas, indicando a maior possibilidade de co-ocorrência entre os conceitos. As cores indicam módulos, ou grupos de conceitos que tendem a aparecer juntos nos tweets.

Figura 1: Rede de conceitos da \#bolsolão

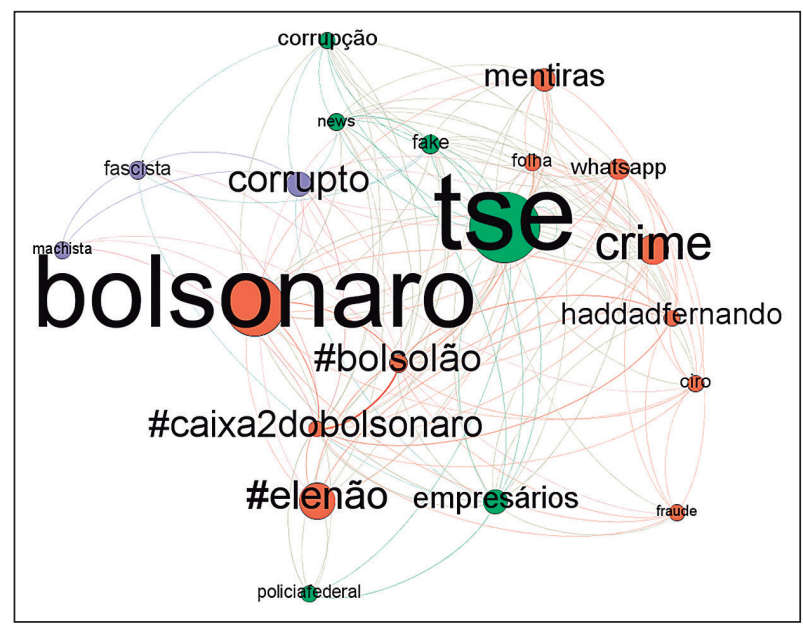

Fonte: Elaboração própria. 
A rede é formada por 20 (vinte) palavras, divididas em 3 (três) agrupamentos, sendo eles nas cores laranja, azul e verde.

O agrupamento laranja interage sobre a prática efetuada na campanha de Jair Bolsonaro ("bolsonaro") à presidência do Brasil, relatada na matéria lançada pela "Folha" de São Paulo (MELLO, 2018), como um crime de "caixa 2", inclusive com o co-impulsionamento de uma hashtag distinta ("\#caixa2dobolsonaro") de modo adjacente à "\#bolsolão". Deste modo, há indícios de que esse discurso a matéria da "Folha" à ocorrência de uma "fraude", a partir do espalhamento de "mentiras" no "whatsapp". Além disso, é possível verificar que, a partir da menção à "\#elenão", o agrupamento laranja estabelece uma articulação comum entre dois candidatos concorrentes nas Eleições de 2018 ("haddadfernando" e "ciro").

O agrupamento compreende o evento como sendo uma prática de "corrupção", voltada a difusão de "fake" "news", a qual alega-se estar ligada à conduta de "empresários". Percebe-se que, nesse agrupamento, as menções à "policiafederal" e ao "TSE" (Tribunal Superior Eleitoral) demonstram que há uma reivindicação à autoridades do campo institucional brasileiro, as quais seriam responsáveis por abordar o caso em uma eventual denúncia de crime ou de ilegalidade.

$\mathrm{O}$ agrupamento azul representa adjetivos utilizados contrariamente a Jair Bolsonaro, através das palavras "machista e "fascista" e "corrupto". Nesse caso, é possível observar a construção de uma imagem negativa acerca de Bolsonaro, de modo que um dos atributos mencionados estaria ligado ao outro, isto é, conectando e aproximando conceitualmente as palavras "machista", "fascista" e "corrupto".

No quadro a seguir (Quadro 2), apresentamos o cálculo dos graus de intermediação de cada um dos conceitos ilustrados no grafo da \#bolsolão.

Quadro 2: Agrupamentos e grau de intermediação dos conceitos da \#bolsolão

\begin{tabular}{|c|c|c|}
\hline Conceito & Agrupamento & Grau de intermediação \\
\hline Tse & Verde & 251.38 \\
\hline Bolsonaro & Laranja & 198.75 \\
\hline \#elenão & Laranja & 93.46 \\
\hline Crime & Laranja & 65.63 \\
\hline Corrupto & Azul & 39.40 \\
\hline Empresários & Verde & 39.37 \\
\hline Mentiras & Laranja & 31.26 \\
\hline Whatsapp & Laranja & 23.57 \\
\hline Fake & Verde & 13.11 \\
\hline \#bolsolão & Laranja & 8.33 \\
\hline
\end{tabular}




\begin{tabular}{|c|c|c|}
\hline Conceito & Agrupamento & Grau de intermediação \\
\hline Machista & Azul & 5.92 \\
\hline Fascista & Azul & 7.82 \\
\hline News & Verde & 4.44 \\
\hline Folha & Laranja & 2.48 \\
\hline Policiafederal & Verde & 2.0 \\
\hline Ciro & Laranja & 0.95 \\
\hline Fraude & Laranja & 0.83 \\
\hline Corrupção & Verde & 0.25 \\
\hline Haddadfernando & Laranja & 0.0 \\
\hline \#caixadobolsonaro & Laranja & 0.0 \\
\hline
\end{tabular}

Fonte: Elaboração própria.

A partir dos graus de intermediação de cada palavra, podemos perceber que os principais conceitos articulados para a constituição do discurso articulado na hashtag \#bolsolão são "TSE", "Bolsonaro" e "\#elenão" (graus 251.38 e 198.75 e 93.46, respectivamente). Isso indica que os principais pontos nodais, articulados como pontes para a formação do discurso presente nesta hashtag, envolvem uma reivindicação ao TSE em relação à sua função que a institucional de investigar "crimes" eleitorais, bem como menções negativas à Jair Bolsonaro, inclusive através de uma hashtag mencionada de forma adjacente (\#elenão).

Na perspectiva da TD (LACLAU, MOUFFE, 2015), é possível observar que a lógica de equivalência presente na constituição dessa hashtag está articulada a partir de duas diretrizes: a) uma demanda, direcionada ao campo institucional brasileiro ("TSE", juntamente com "policiafederal"), para que "bolsonaro" e "empresários" sejam investigados (módulo verde); b) a constituição de Jair Bolsonaro como um inimigo em comum ("\#elenão"), frente ao contexto de campanha eleitoral daquele momento, sob uma noção de que evitar a vitória de Bolsonaro - um candidato "machista", "fascista" e "corrupto" - seria mais importante do que eleger candidato um "x" ou "y" (módulos laranja e azul).

A lógica da diferença (LACLAU; MOUFFE, 2015) mostra-se presente, principalmente, na construção de uma articulação entre dois candidatos concorrentes "haddadfernando" e "ciro", sob um mesmo discurso. Portanto, tal como expõe a métrica da modularidade, a emergência da \#bolsolão serviu como um "cimento ideológico" propício à formação de uma coalizão (LACLAU, 2005, p.237). Dessa forma, por meio da conexão entre diversas palavras, tais como "TSE", "corrupto", "empresários", "mentiras", "fascista", "machista" e "fraude" - além da própria hashtag "\#elenão" -, tornou-se possível que interagentes identificados com 
candidatos concorrentes entre si formassem uma "aliança", articulada por uma negação em comum à ideia de Jair Bolsonaro como presidente.

Na figura seguinte (Figura 2), observamos o grafo e os principais tweets relativos às redes de interação que se articularam favoravelmente a Jair Bolsonaro.

Figura 2: Rede de conceitos da \#marqueteirosdojair

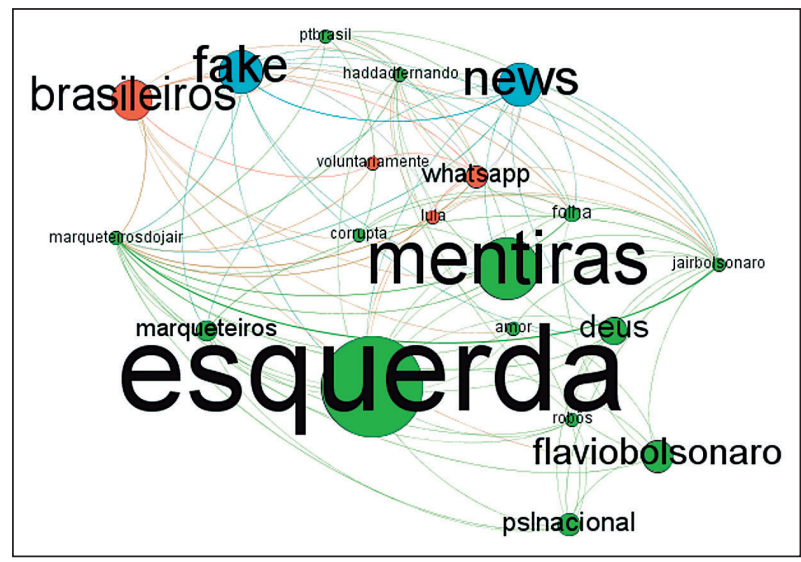

Fonte: Elaboração própria.

A rede atinente à \#marqueteirosdojair é formada por 20 (vinte) palavras, separadas em 3 (três) agrupamentos, estando representados nas seguintes cores: verde, azul e laranja.

O agrupamento verde concentra a maior parte das interações, orientadas sob a ideia de que a matéria lançada pela Folha de São Paulo (MELLO, 2018) ("folha") concerne a "mentiras" de autoria da "esquerda" "corrupta". É interessante destacar que a notícia publicada pelo veículo tradicional de mídia passa a ser interpretado como mentiroso, o que de certa forma demonstra um descrédito ao jornalismo profissional, que acaba sendo marginalizado no ambiente político polarizado (MIGUEL, 2019). Juntamente a isso, tendo em vista o período de campanha eleitoral, a alegação de que a notícia corresponde a uma informação falsa passa a ser vista sob o contexto do atentado da facada contra Jair Bolsonaro, através de uma narrativa na qual o, então candidato, apenas estaria vivo por um milagre de "Deus".

O grupo laranja traz a ideia de que os disparos de conteúdo realizados em grupos de WhatsApp são efetuados autenticamente, não sendo, portanto, efetuados por robôs, mas por apoiadores identificados como "brasileiros", os quais voluntariamente contribuem na campanha de Jair Bolsonaro. Além disso, há uma menção negativa à figura de "Lula" - a despeito de ele não ser candidato na Eleição de 2018 -, no sentido de que o ex presidente, diferentemente de Bolsonaro, não possui grupos de apoiadores com tamanho nível de engajamento. 
O agrupamento azul está concentrado, unicamente, na noção de que o evento em questão corresponde a uma "fake" "news", isto é, a uma notícia de caráter falso.

A seguir o Quadro 3 contendo os conceitos e os respectivos graus de intermediação.

Quadro 3: Grau de intermediação entre os conceitos da \#marqueteirosdojair

\begin{tabular}{|c|c|c|}
\hline Conceito & Agrupamento & Grau de intermediação \\
\hline Esquerda & Verde & 16.16 \\
\hline Mentiras & Verde & 9.0 \\
\hline News & Azul & 5.5 \\
\hline Fake & Azul & 5.5 \\
\hline Brasileiros & Laranja & 5.0 \\
\hline Flaviobolsonaro & Verde & 3.33 \\
\hline Deus & Verde & 2.58 \\
\hline Pslnacional & Verde & 1.75 \\
\hline Whatsapp & Laranja & 1.5 \\
\hline Marqueteiros & Verde & 1.33 \\
\hline Folha & Verde & 0.33 \\
\hline Ptbrasil & Verde & 0.0 \\
\hline Voluntariamente & Laranja & 0.0 \\
\hline Robôs & Verde & 0.0 \\
\hline Corrupta & Verde & 0.0 \\
\hline Lula & Laranja & 0.0 \\
\hline Haddadfernando & Verde & 0.0 \\
\hline Jairbolsonaro & Verde & 0.0 \\
\hline \#marqueteirosdojair & Verde & 0.0 \\
\hline Robôs & Verde & 0.0 \\
\hline \#caixadoisdobolsonaro & Verde & \\
\hline
\end{tabular}

Fonte: Elaboração própria.

As palavras da \#marqueteirosdojair que se destacam pela maior proeminência dos graus de intermediação são "esquerda" e "mentiras" (graus 16.16 e 9.0, respectivamente). Isso demonstra que essas palavras, manifestamente negativas em 
relação ao "outro inimigo", foram os principais pontos nodais articulados nesta hashtag e, portanto, responsáveis por encadear em um só discurso os diferentes conceitos observados no grafo.

Constatamos, dessa forma, que a lógica de equivalência que orienta a emergência dessa hashtag está atrelada à demarcação da "esquerda" como o inimigo em comum, responsável pelo constante espalhamento de "mentiras" sobre Jair Bolsonaro. Além disso, destacamos a autoidentificação dos interagentes da \#marqueteirosdojair como "brasileiros" (grau de intermediação 5.0), no sentido de que a possibilidade de constituição de uma determinada nacionalidade infere, necessariamente, a negação de outras. Assim, o termo "brasileiros" envolve uma identificação positiva, por um lado, e negativa, por outro, isto é, para que um seja brasileiro, um "outro" não pode ser - isso, segundo Laclau (2014, p.106), está associado à dinâmica pela qual uma articulação discursiva é constituída, em negação a um outro, uma vez que "a presença de um inimigo me impede de eu constituir a minha própria identidade".

Quanto à lógica da diferença (LACLAU; MOUFFE, 2015), não se observa nessa hashtag, conforme a exposição dos principais conceitos mencionados nas interações, uma articulação entre identidades que, no contexto identificado, mostram-se notadamente divergentes. Tal como definido pelo grau da modularidade, a rede de interações dessa hashtag mostra-se relativamente condensada, visto que das 20 (vinte) palavras que compõem a hashtag, 15 (quinze) delas estão atreladas apenas a um agrupamento (verde) - o que indica um alto grau de coesão entre as interações. De todo modo, os elementos discursivamente articulados para a formação da \#marqueteirosdojair abrangem ideais religiosos ("Deus"), a dedicação voluntária ("voluntariamente") de "brasileiros" à candidatura de Jair Bolsonaro, apoiadores do partido "PSL" (Partido Social Liberal) e afetos negativos direcionados à figura de inimigos ("Lula" e "haddadfernando").

Comparativamente, constatamos nas duas hashtags a emergência de uma dinâmica relacional de confronto, pela qual se formaram as articulações discursivas observadas. $\mathrm{Na}$ \#bolsolão, notamos que o "exterior constitutivo" (LACLAU; MOUFFE, 2015) em questão - isto é, o inimigo em comum contra o qual um discurso se articula - trata-se de Jair Bolsonaro. Isso pode ser observado, inclusive, a partir da coalizão implícita na hashtag \#elenão, composta entre interações identificadas a candidatos concorrentes nas Eleições de 2018. Já na \#marqueteirosdojair, observamos como a "esquerda" figurou como a identidade contra a qual se articularam as interações em apoio à campanha de Jair Bolsonaro. Diante desses aspectos, observamos na nossa análise que essa dinâmica antagônica tende a produzir, no social, visões contraditórias acerca dos significados de um mesmo objeto - constituindo, portanto, um objeto de disputa. 
Nesse caso, isso pode ser demonstrado ao se verificar a presença dos conceitos "fake", "news" e "corrupção" em ambas as hashtags. No tocante a isso, observamos que, em cada hashtag, tanto o significado de "fake news", como "corrupção", são construídos de modo objetivamente contraditórios, como se cada discurso representasse um lado de uma mesma moeda.

Em um destes lados, na \#marqueteirosdojair, o significado de "fake news" está relacionado a supostas notícias falsas publicadas por inimigos de Jair Bolsonaro ("esquerda"), sendo, mais especificamente, o jornal Folha de São Paulo, veículo de mídia tradicional no Brasil, colocado em xeque nesse cenário. Do mesmo modo, o conceito de "corrupção" também atrelado ao campo identificado nas interações como da "esquerda", da qual entendem praticar o "ptbrasil", ou seja, o Partido dos Trabalhadores (PT).

No outro lado, na \#bolsolão, observamos a perspectiva inversa, visto que tanto a prática de "fake news" quanto de "corrupção" está atrelada à figura de Jair Bolsonaro ("Bolsonaro"), bem como a de "empresários", uma vez que ambos são vistos como responsáveis por um "crime", relacionado à prática de disparos de mensagens no WhatsApp, tal como relatada na matéria publicada.

Assim, ao se observar esse tipo de dinâmica nas interações no Twitter, tornase verossímil a constatação do aspecto primário de não-racionalidade, atinente às dinâmicas sociais contemporâneas (LACLAU, 2005). Nessa ótica, os aportes da TD permitem observar uma predominância afetiva-valorativa no teor das interações sociopolíticas no digital, em detrimento de uma lógica retórica/argumentativa (LACLAU, 2005).

No sentido constatado por Laclau e Mouffe (2015, p.179), a relacionalidade necessária à formação de toda identidade prescinde do fato de que "todos os valores são valores de oposição e são definidos unicamente por sua diferença". Nesse sentido, configura-se uma tendência de que, diante da crescente complexidade do social contemporâneo, os significados dos fenômenos e dos conceitos tendem a encontrar os seus limites objetivos nas contradições advindas da formação de antagonismos discursivos.

A observação das interações no Twitter, portanto, permite a identificação dos limites da "ordem social", vista sob a noção de que existe uma única "sociedade", integrada a partir do consenso. Tal como propôs Jodi Dean (2013), é possível verificar uma correspondência entre as características das interações sociais constituídas por meio da internet e conceitos vinculados à abordagem da TD. Na medida em que o social vem a ser, cada vez mais, um fenômeno concebido mediante o constante no intercâmbio on/off-line, a percepção sobre a realidade perpassa pelas características específicas relativas às relações estabelecidas em plataformas digitais (VAN DIJCK, 2013). Nesse caso, tendo em vista a nossa análise, a observação das interações no Twitter favorece a compreensão de que não existe uma sociedade 
formada por uma “ordem objetiva”, mas um social contingente e precário, formado a partir de antagonismos que representam constantemente os limites da dita "sociedade" (LACLAU, 2014, p.112).

As hashtags corresponderiam, dessa forma, a pontos nodais, através dos quais uma heterogeneidade de elementos no social pode ser articulada em combate a um mesmo inimigo (LACLAU; MOUFFE, 2015). Conforme entende Papacharissi (2009), a linha tênue entre o que é público e o que é privado no âmbito das interações no Twitter favorece, por um lado, a emergência de mensagens narcísicas e/ou segregadas para um determinado grupo, enquanto, por outro, a possibilidade de que essas mesmas mensagens sejam comunicadas para um público maior. Assim, no sentido da terminologia dos estudos em análise de redes sociais, é possível considerar que uma hashtag poderia representar um ponto nodal quando há nela uma dinâmica de homofilia, isto é, de um agrupamento de interações caracterizado pelo alto grau de similaridade compartilhado entre os nós, indicando a produção de uma redundância informacional (RECUERO, 2017).

Em uma perspectiva mais abrangente, entendemos que a abordagem teórico-metodológica proposta neste trabalho privilegia a observação do contexto de polarização política no Brasil como um fenômeno dinâmico, contingente e contraditório no social. Consoante a isso, a dinamicidade se dá pelo fato de que o contexto de disputa em torno de um objeto - no caso analisado, a totalidade de um discurso referente à matéria lançada pelo jornal - é constituído em relação a eventos específicos, os quais acontecem a todo instante no social. Nesse sentido, a contingência decorre do aspecto de que redes e contextos polarizados de disputa emergem a partir da formação de coalizões circunstanciais, cada qual articulada na direção de combater um inimigo em comum (LACLAU, 2005). Assim, conforme verificamos nas hashtags, a contradição entre discursos passa a ser uma característica expressiva de um social contemporâneo caracterizado por processos permanentes de disputas antagônicas em torno de um objeto impossível: a possibilidade - ou a hegemonia, conforme os termos da TD (LACLAU; MOUFFE, 2015) - de significar totalmente a sociedade. Todavia, constatamos que a nossa abordagem mostrou-se limitada no que toca à observação do provável aspecto de precariedade, relativa à polarização política no contexto político-eleitoral brasileiro, uma vez que, para isso, seriam necessárias análises comparativas entre diferentes hashtags antagônicas atinentes a eventos distintos.

No sentido de uma contribuição à Sociologia Digital, a nossa abordagem envolve um esforço voltado à proposição de um método de interface, tal como sugerido por Marres (2017), bem como aos diferentes objetivos desta subdisciplina, descritos por Lupton (2015). Primeiramente, mediante a utilização de métodos e de ferramentas digitais, buscamos a construção de uma "oportunidade epistêmica" (MARRES, 2017) capaz de observar e responder questionamentos socialmente 
pertinentes. Em um segundo momento, ao analisarmos hashtags no Twitter como discursos articulados digitalmente, refletimos de que forma os recursos de plataformas digitais são apropriados para reproduzir - ou mesmo potencializar problemáticas constituídas sob um intercâmbio on/off-line constante. Por fim, ao aproximarmos os aportes metodológicos da ARS à lente teórico-epistemológica da TD de Laclau e Mouffe (2015), refletimos sobre como teorias sociais já consolidadas podem, simultaneamente, aprofundar e serem aprofundadas a partir da investigação de aspectos quantitativos e qualitativos presentes nas interações sociais digitais.

\section{Considerações Finais}

O presente trabalho abordou a formação de articulações discursivas no Twitter, no contexto político-eleitoral polarizado, das Eleições de 2018 no Brasil. A partir de uma perspectiva interdisciplinar, combinando os aportes teóricoepistemológicos da Teoria do Discurso aos aportes metodológicos da Análise de Redes Sociais na internet, o trabalho analisou interações sociais desempenhadas por meio de plataformas digitais, trazendo uma contribuição teórico-metodológica para a Sociologia Digital.

Assim analisamos as articulações discursivas formadas nas hashtags "\#bolsolão" e "\#marqueteirosdojair", as quais emergiram antagonicamente uma à outra, como discursos organizados em disputa, no contexto do lançamento da matéria do jornal Folha de São Paulo, do dia 18 de outubro de 2018, "Empresários bancam campanha contra o PT pelo WhatsApp" (MELLO, 2018). A partir disso, identificamos os elementos articulados na formação de cada uma das duas hashtags analisadas, descrevendo as equivalências e quais as diferenças verificadas em cada uma delas. Dessa forma, apresentamos a emergência de antagonismos discursivos, principalmente em um contexto sociopolítico brasileiro constatado como polarizado, como eventos associados a contradições no social, as quais expõem os limites objetivos atinentes ao discurso.

A originalidade apresentada neste trabalho, dentro da proposta da Sociologia Digital, abrange duas diretrizes. Por um lado, a proposição de uma base teóricometodológica voltada à investigação da polarização política e/ou da formação de antagonismos discursivos em interações digitais. Por outro, a reflexão a respeito da aplicabilidade de teorias e métodos já existentes ao empírico observado digitalmente, utilizando-se inclusive de ferramentas disponíveis digitalmente, como forma de oportunizar investigações de caráter quantitativo e qualitativo.

Por fim, consideramos que as limitações da nossa proposta podem inspirar o desenvolvimento de trabalhos futuros. Assim, apontamos tanto para a possibilidade de maior aprofundamento teórico-epistemológico em relação à complexidade das 
Antagonismos discursivos nas hashtags \#marqueteirosdojair e \#bolsoläo no Twitter nas eleiçōes de 2018 no Brasil: contribuiçôes da análise de redes sociais à sociologia digital

articulações discursivas efetuadas digitalmente, quanto para a proposição de novas possibilidades metodológicas envolvendo uma articulação entre Análise de Redes Sociais e teorias sociológicas dispostas a compor o escopo da Sociologia Digital.

\title{
DiscuRsive ANTAGONISMS ON THE HASHTAGS \#marqueteirosdojaIr and \#bolsoläo on TwitTer IN THE BRAZILIAN PRESIDENTIAL ELECTION OF 2018: CONTRIBUTIONS FROM THE SOCIAL NETWORK ANALYSIS TO THE DIGITAL SOCIOLOGY
}

\begin{abstract}
This article aims to analyze the configuration of discursive antagonisms in the hashtags \#bolsolão and \#marqueteirosdojair during the context of the 2018 presidential elections in Brazil. In contribution to the theoretical and methodological challenges of the Digital Sociology, we started from an interdisciplinary perspective, approaching Ernesto Laclau and Chantal Mouffe's Theory of Discourse to the Social Network Analysis on the internet. We collected a total of 26,548 tweets from the Twitter API through the software NodeXL Pro. Thus, the two hashtags selected were investigated using a contingency analysis, according to the metrics of modularity and degree of intermediation. By doing so, we verified the elements linked to the formation of the two hashtags, describing how they are articulated according to the logics of equivalence and difference. In conclusion, our theoretical-methodological proposal enables the observation of the political polarization as a dynamic, contingent and socially contradictory phenomenon, associated with the emergence of discursive antagonisms.
\end{abstract}

KEYWORDS: Digital Sociology. Discourse Theory. Twitter. Social Network Analysis. Brazilian elections of 2018.

\section{Antagonismos discuRsivos en lOS hashtags \#MARQUETEIROSDOJAIR Y \#BOLSOLÃO NO TWITTER EN LAS ELECCIONES DE 2018 EN BRASIL: CONTRIBUCIONES DEL ANÁLISIS DE REDES SOCIALES A LA SOCIOLOGÍA DIGITAL}

RESUMEN: Este artículo pretende analizar la formación de antagonismos discursivos en los hashtags \#bolsolão y \#marqueteirosdojair, en el contexto polarizado de las elecciones de 2018 en Brasil. En la contribución a los desafíos teórico-metodológicos de la Sociología Digital, partimos de un enfoque 
interdisciplinario, llevando la Teoría del Discurso de Ernesto Laclau y Chantal Mouffe al Análisis de las Redes Sociales en Internet. A partir de esto, recogimos un total de 26.548 tweets del API de Twitter a través del software NodeXL Pro. Por lo tanto, investigamos los dos hashtags seleccionados a través de un análisis de contingencia, de acuerdo con la métrica de la modularidad y el grado de intermediación. De esta manera, verificamos qué sentidos están encadenados en la formación de los dos hashtags, describiendo cómo se articulan según las lógicas de equivalencia y diferencia. Llegamos a la conclusión de que nuestra propuesta teórico-metodológica permite observar la polarización política como un fenómeno dinámico, contingente y contradictorio en la sociedad, asociado a la aparición de antagonismos discursivos.

PALABRAS CLAVE: Sociología Digital. Teoría del Discurso. Twitter. Análisis de Redes Sociales. Elecciones 2018.

\section{REFERÊNCIAS}

BARDIN, Laurence. Análise de Conteúdo. Lisboa: Edições 70, 1977.

BENKLER, Yochai; FARIS, Robert; ROBERTS, Hal. Network Propaganda: manipulation, disinformation, and radicalization in american politics. New York: Oxford University Pres, 2018.

BLONDEL, Vincent; GUILLAUME, Jean-Loup; LAMBIOTTE, Renaud; LEFEBVRE, Etienne. Fast unfolding of communities in large networks. Journal of Statistical Mechanics: Theory and Experiment, (10), P1000, 2008.

BOYD, Danah. ELLISON, Nicole. Social network sites: Definition, history, and scholarship. Journal of Computer-Mediated Communication, 13(1), article 11, 2007. Disponível em: https://doi.org/10.1111/j.1083-6101.2007.00393.x. Acesso em 10/09/2019.

BRUNS, Axel. Are Filter Bubbles Real? Cambridge: Polity Press, 2019.

BRUNS, Axel \& BURGESS, Jean E. The use of Twitter hashtags in the formation of ad hoc publics. Paper presented at 6th European Consortium for Political Research General Conference, August 25-27, 2011. University of Iceland, Reykjavik. Disponível em: http:// eprints.qut.edu.au/46515/. Acesso em: 28 jun. 2020.

BURITY, Joanildo Albuquerque. Discurso, política e sujeito na teoria da hegemonia de Ernesto Laclau. In: MENDONÇA, Daniel de; RODRIGUES, Léo Peixoto. PósEstruturalismo e Teoria do Discurso: em torno de Ernesto Laclau. 2. ed. Porto Alegre: EDIPUCRS, 2014. 
Antagonismos discursivos nas hashtags \#marqueteirosdojair e \#bolsoläo no Twitter nas eleiçōes de 2018 no Brasil: contribuiçôes da análise de redes sociais à sociologia digital

CESARINO, Letícia. Como vencer uma eleição sem sair de casa: a ascensão do populismo digital no Brasil. Internet \& Sociedade, v. 1, n. 1, 2020, p.91-120. Disponível em: https:// revista.internetlab.org.br/serifcomo-vencer-uma-eleicao-sem-sair-de-casa-serif-a-ascensaodo-populismo-digital-no-brasil/. Acesso em: 3 mar. 2020.

COSTA, Everton Garcia da. As Manifestações no Brasil vistas à luz da Teoria do Discurso. Revista Sociologia em Rede, v. 4, n. 4, 2014, p.15-36. Disponível em: http://redelp.net/ revistas/index.php/rsr/article/view/2costa4/132. Acesso em: 29 out. 2019.

COSTA, Everton Garcia da; FREITAS, Felipe Corral de; MERLIN, Nora. Apresentação do Dossiê "Teoria do Discurso e seu alcance interdisciplinar e transversal: explorando debates empíricos e teóricos. Simbiótica, v. 6, n. 2, 2019. Disponível em: https://periodicos.ufes.br/ simbiotica/article/view/28397/20244. Acesso em: 30 mai. 2020.

DEAN, Jodi. Society doesn't exist. First Monday. Chicago, v. 18, n. 3. mar, 2013. Disponível em: http://firstmonday.org/ojs/index.php/fm/article/view/4616/3419. Acesso em: 28 set. 2019.

FARKAS, Johan; SCHOU, Jannick; NEUMAYER, Christina. Platformed antagonism: racist discourses on fake Muslim Facebook pages, Critical Discourse Studies, v. 15, n. 5, 2018, p.463-480. Disponível em: https://doi.org/10.1080/17405904.2018.1450276. Acesso em: 29 set. 2019.

FRAGOSO, Suely; RECUERO, Raquel; AMARAL, Adriana. Métodos de pesquisa para internet. $4^{\mathrm{a}}$ reimpressão. Porto Alegre, Sulina, 2016.

GERBAUDO, Paolo. Social media and populism: an elective affinity? Media, Culture \& Society, v. 40, n 5, 2018, p.1-9. Disponível em: https://doi.org/10.1177/0163443718772192. Acesso em: 02 mar. 2020.

GILLESPIE, Tarleton. The politics of 'platforms'. New Media \& Society, v. 12, n. 3, p.347364, 2010. Disponível em: https://ssrn.com/abstract=1601487. Acesso em: 01 mar. 2020.

HABERMAS, Jürgen. Consciência Moral e Agir Comunicativo. Rio de Janeiro: Tempo Brasileiro, 1989.

HERRING, Susan. Computer-mediated discourse. In: TANNEN, Deborah; HAMILTON, Heidi; SCHIFFRIN, Deborah (Org.). Handbook of discourse analysis. Oxford: Blackwell, 2001.

LACLAU, Ernesto. The Rhetorical Foundations of Society. London: Verso, 2014.

LACLAU, Ernesto. La razón populista. Buenos Aires: Fondo de Cultura Económica, 2005.

LACLAU, Ernesto; MOUFFE, Chantal. Hegemonia e Estratégia Socialista. São Paulo: Intermeios, 2015. 
LUPTON, Deborah. Digital Sociology. London and New York: Routledge, 2015.

MARRES, Noortje. Digital Sociology: The Reinvention of Social Research. Cambridge: Polity Press, 2017.

MARRES, Noortje; GERLITZ, Carolin. Interface Methods: Renegotiating Relations between Digital Social Research, STS and Sociology. The Sociological Review, 64(1), 2016, p.21-46. Disponível em: https://doi.org/10.1111/1467-954X.12314. Acesso em: 01 mar. 2020.

MELLO, Patrícia Campos. Empresários bancam campanha contra o PT pelo WhatsApp. FOLHA DE S.PAULO. Publicado em de 18 outubro de 2018. Disponível em: https://www1. folha.uol.com.br/poder/2018/10/empresarios-bancam-campanha-contra-o-pt-pelo-whatsapp. shtml. Acesso em: 28 jun. 2020.

MIGUEL, Luis Felipe. Jornalismo, polarização política e a querela das fake news. Estudos em Jornalismo e Mídia, v. 16, n. 2, p. 46-58, 2019.

MISKOLCI, Richard. Sociologia Digital: notas sobre pesquisa na era da conectividade. Contemporânea, v. 6, n. 2, jul-dez, 2016. Disponível em: http://www.contemporanea.ufscar. br/index.php/contemporanea/article/view/525. Acesso em: 27 fev. 2020.

MOUFFE, Chantal. Quais espaços públicos para práticas de arte crítica? Arte \& Ensaios 27, ano XXI, n. 27, 2013, p.181-199. Disponível em: http://www.ppgav.eba.ufrj.br/wp-content/ uploads/2015/03/tematicas-chantal.pdf. Acesso em: 15 set. 2019.

MOUFFE, Chantal. Por um modelo agonístico de democracia. Revista de Sociologia e Política, n. 25, p.11-23, nov, 2005. Disponível em: http://www.scielo.br/pdf/rsocp/ n25/31108.pdf. Acesso em: 29 set. 2019.

NASCIMENTO, Leonardo Fernandes. A Sociologia Digital: um desafio para o Século XXI. Sociologias: Porto Alegre, ano 18, n. 41, 2016. p.216-241. Disponível em: http://seer.ufrgs. br/index.php/sociologias/article/view/53754/37173. Acesso em: 15 jun. 2018.

NEWMAN, Mark. Networks: An Introduction. New York: Oxford University Press, 2010.

OSGOOD, Charles. The representational model and relevant research methods. In: I. de S. Pool (ed.). Trends in Content Analysis. Champaign, Ill: University of Illinois Press, 1959.

PAPACHARISSI, Zizi. Without You, I'm Nothing: Performances of the self on Twitter. International Journal of Communication, v. 6, 2012, p.1989-2006. Disponível em: https:// ijoc.org/index.php/ijoc/article/view/1484/775. Acesso em: 29 set. 2019.

PAPACHARISSI, Zizi. The virtual sphere 2.0: The Internet, the public sphere, and beyond. In: CHADWICK, Andrew; HOWARD, Philip. Routledge handbook of Internet politics. Abingdon: Routledge, 2009. 
Antagonismos discursivos nas hashtags \#marqueteirosdojair e \#bolsoläo no Twitter nas eleiçôes de 2018 no Brasil: contribuiçôes da análise de redes sociais à sociologia digital

PARISER, Eli. The Filter Bubble: What The Internet Is Hiding from You. London: Penguin, 2011.

RECUERO, Raquel. Estudando Discursos em Mídia Social: uma proposta metodológica. In: SILVA, Tarcizo; BUCKSTEGGE, Jaqueline; ROGEDO, Pedro. Estudando cultura e comunicação com mídias sociais. Brasília: IBPAD, 2018.

RECUERO, Raquel. Introdução à análise de redes sociais. Salvador: EDUFBA, 2017. Disponível em: https://repositorio.ufba.br/ri/handle/ri/24759. Acesso em: 27 jun. 2020.

RECUERO, Raquel.; BASTOS, Marco.; ZAGO, Gabriela. Análise de Redes para Mídia Social. Porto Alegre: Sulina, 2015.

SALAZAR, Eduardo. Hashtags 2.0 - An Annotated History of the Hashtag and a Window to its Future. Icono 14, v. 15, n. 2, p.16-54. Disponível em: https://dialnet.unirioja.es/descarga/ articulo/6054356.pdf. Acesso em: 04 jul. 2020.

SELWYN, Neil. What is Digital Sociology? Cambridge: Polity Press, 2019.

SOARES, Felipe; RECUERO, Raquel; ZAGO, Gabriela. Asymmetric Polarization on Twitter and the 2018 Brazilian Presidential Elections. Proceedings of the International Conference on Social Media \& Society, 2019. Disponível em: https://doi.org/10.1145/3328529.3328546. Acesso em: 03 mar. 2020.

VAN DIJCK, José. The Culture of Connectivity. Oxford: Oxford University Press, 2013.

WASSERMAN, Stanley.; FAUST, Katherine. Social Network Analysis: methods and aplications. Cambridge: Cambridge University Press, 1994.

WYNN, Jonathan. Digital sociology: emergent technologies in the field and the classroom. Sociological Forum, v. 24, n. 2, 2009, p.448-456. Disponível em: https://doi.org/10.1111/ j.1573-7861.2009.01109.x. Acesso em: 27 fev. 2020.

Recebido em 10/03/2020.

Aprovado em 06/05/2020. 
